\title{
Design and Analysis of a Continuous and Non-Invasive Multi-Wavelength Optical Sensor for Measurement of Dermal Water Content
}

\author{
Mohammad Mamouei ${ }^{1,2, *(\mathbb{D}) \text {, Subhasri Chatterjee }}{ }^{2}$, Meysam Razban ${ }^{2}$, Meha Qassem ${ }^{2}$ \\ and Panayiotis A. Kyriacou 2 (D)
}

1 Deep Medicine, Oxford Martin School, University of Oxford, Oxford OX1 2JD, UK

2 Research Centre for Biomedical Engineering, City University of London, London EC1V 0HB, UK; Subhasri.Chatterjee.2@city.ac.uk (S.C.); meysam.razban.1@city.ac.uk (M.R.); Meha.Qassem@city.ac.uk (M.Q.); P.Kyriacou@city.ac.uk (P.A.K.)

* Correspondence: Mohammad.Mamouei@wrh.ox.ac.uk

$+\quad$ This paper is an extended version of our paper published in: Mamouei, M.; Qassem, M.; Razban, M.; Kyriacou, P.A. Measurement of dermal water content using a multi-wavelength optical sensor. In Proceedings of the 2020 42nd Annual International Conference of the IEEE Engineering in Medicine \& Biology Society (EMBC), Virtual Conference, 20-24 July 2020; pp. 4353-4356.

Citation: Mamouei, M.; Chatterjee,

S.; Razban, M.; Qassem, M.; Kyriacou,

P.A. Design and Analysis of a

Continuous and Non-Invasive Multi-Wavelength Optical Sensor for Measurement of Dermal Water Content. Sensors 2021, 21, 2162. https://doi.org/10.3390/s21062162

Academic Editor: Benoit Piro

Received: 16 February 2021

Accepted: 16 March 2021

Published: 19 March 2021

Publisher's Note: MDPI stays neutral with regard to jurisdictional claims in published maps and institutional affiliations.

Copyright: (c) 2021 by the authors. Licensee MDPI, Basel, Switzerland. This article is an open access article distributed under the terms and conditions of the Creative Commons Attribution (CC BY) license (https:// creativecommons.org/licenses/by/ $4.0 /)$.

\begin{abstract}
Dermal water content is an important biophysical parameter in preserving skin integrity and preventing skin damage. Traditional electrical-based and open-chamber evaporimeters have several well-known limitations. In particular, such devices are costly, sizeable, and only provide arbitrary outputs. They also do not permit continuous and non-invasive monitoring of dermal water content, which can be beneficial for various consumer, clinical, and cosmetic purposes. We report here on the design and development of a digital multi-wavelength optical sensor that performs continuous and non-invasive measurement of dermal water content. In silico investigation on porcine skin was carried out using the Monte Carlo modeling strategy to evaluate the feasibility and characterize the sensor. Subsequently, an in vitro experiment was carried out to evaluate the performance of the sensor and benchmark its accuracy against a high-end, broad band spectrophotometer. Reference measurements were made against gravimetric analysis. The results demonstrate that the developed sensor can deliver accurate, continuous, and non-invasive measurement of skin hydration through measurement of dermal water content. Remarkably, the novel design of the sensor exceeded the performance of the high-end spectrophotometer due to the important denoising effects of temporal averaging. The authors believe, in addition to wellbeing and skin health monitoring, the designed sensor can particularly facilitate disease management in patients presenting diabetes mellitus, hypothyroidism, malnutrition, and atopic dermatitis.
\end{abstract}

Keywords: skin hydration; optical sensor; near infrared spectroscopy; Monte Carlo simulation

\section{Introduction}

Dermal water content is an important biophysical parameter that is interrelated with epidermal barrier function [1,2] and the desquamation process [1,3]. At the molecular level, water structure and bond formation also influence skin attributes such as dermal elasticity [4,5] and skin aging where water binding has been found to change into a tetrahedron form over time [6,7]. The skin's outer layer, known as the stratum corneum (SC), acts as a primary barrier to the external environment, and is responsible for regulating the rate of water evaporation through skin and its water-retaining capabilities $[3,8]$.

Measurements of dermal water content are often essential in both dermatological and cosmetic investigations, and can be carried out using a variety of non-invasive techniques. Most common are electrical capacitance measurements of skin moisture [9,10], and assessment of skin barrier function through closed-chamber instruments designed to measure 
transepidermal water loss (TEWL). However, both techniques are susceptible to environmental influences [9], have an indirect relationship with the parameter of interest (and hence provide arbitrary outputs), and can lack sensitivity, which as a result, can produce erroneous readings [11-13]. In addition, such devices usually require several minutes of equilibration time in order to stabilize. Alternative techniques such as Raman and attenuated total reflectance (ATR) spectroscopy, and confocal Raman microscopy (CRM) have also been employed for the analysis of skin hydration and permit non-invasive assessment of multiple skin parameters. CRM, in particular, is characterized by high measurement accuracy and the ability to obtain detailed depth profiles of water distribution within the SC layer and hydrogen bonding state of water molecules [14-16]. However, despite the advantages of such techniques, these are often large, expensive, commercially unavailable, and their outputs can be difficult to interpret.

In recent years, we have employed near-infrared (NIR) spectroscopy in comprehensive analysis of skin hydration in the optical window of 750-2500 nm, with results demonstrating a clear capability and accuracy of the technique for estimation of dermal water content. Efforts are now focused on the design and development of a transcutaneous sensor based on NIR technology that is specific to measurement of skin hydration. NIR was the preferred choice of method because absorption bands of water, proteins, and lipids inside the skin are visible in NIR spectra, and can be used to deduce information pertaining to various aspects of skin health such as hydration [17-25], and can differentiate between bound and free types of water inside the skin [18]. In turn, this allows a more precise method of measurement, and an added advantage is that NIR spectroscopic instrumentation can easily be equipped with fiber optic probes for in vivo measurements at various anatomical sites. Although there has been interest in improving this technique for the purpose of skin hydration measurements [26,27], a reliable handheld or portable measuring instrument of this type does not yet exist.

From the literature [18] and our previous work [28], it is known that a typical NIR spectrum of skin, such as that shown in Figure 1, is dominated by two bands around 1450 and $1900 \mathrm{~nm}$, attributed to the combination of $\mathrm{OH}$ and $\mathrm{HOH}$ vibrations of water, and additional bands around 1200 and $970 \mathrm{~nm}$ that also relate to dermal water. This property can be exploited to build a multi-wavelength optical sensor that emits light at the wavelengths of interest, and thereby eliminate the necessity of using benchtop instruments with broad lamp sources and the generation of wide range spectra. NIR spectra of porcine skin also include absorption bands relating to $\mathrm{CH}$ and $\mathrm{NH}$ functional groups from lipid and protein constituents of skin. These include overtones of alkyl $\mathrm{CH}$ groups in skin lipids at around 1760 and $1730 \mathrm{~nm}$, and a combination band from the same group around $2300 \mathrm{~nm}[18,28]$. Bands attributed to $\mathrm{NH}$ bonding are typically observed at 2050 and $1500 \mathrm{~nm}$, with a further $\mathrm{C}=\mathrm{O}$ combination band at $2180 \mathrm{~nm}$, as well as a shoulder-like character around $2000 \mathrm{~nm}$ representative of bound water $[18,20,28]$.

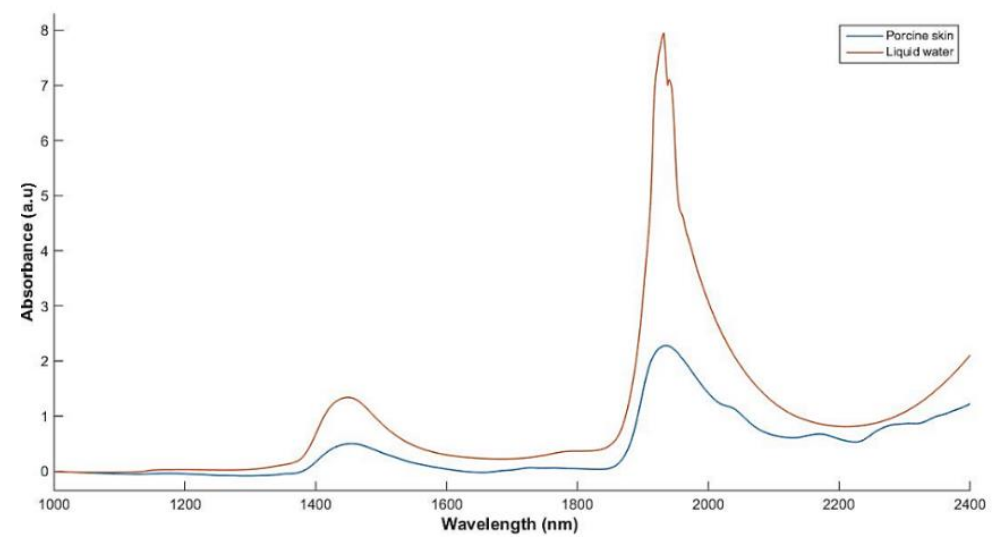

Figure 1. A typical NIR spectrum of liquid water versus that of porcine skin. 
Given the characteristics highlighted above, this paper reports on the development and evaluation of a digital multi-wavelength optical sensor that performs continuous and non-invasive measurement of dermal water content. The sensor was designed to detect optical signals at four wavelengths in the NIR region that relate to skin hydration, and was evaluated against both gravimetric and spectrophotometric measurements in a desorption experiment, with multivariate techniques being applied for data analysis and interpretation.

In order to evaluate and standardize an optical sensor, it is of utmost importance to understand the underlying light-tissue interactions pertaining to the sensor geometry (i.e., wavelengths, shape and size of the optical source and detector, source-detector separation, etc.) and the tissue region of interest (ROI). The sensor performance relies on the wavelength-dependent scattering and absorption properties of the ROI, which varies with tissue water content. Thus, for the purpose of providing comprehensive analysis, a robust model of light-tissue interaction was developed and evaluated using the Monte Carlo method. Monte Carlo is a well-known method used to simulate light-tissue interactions and produces accurate and reliable outcomes [29]. Though several Monte Carlo investigations have been carried out with different human skin ROI, no published literature is found on Monte Carlo simulation with porcine skin in relation to skin hydration. This in silico analysis, along with the aforementioned in vitro evaluation, provides a useful tool in the design and optimization of novel optical skin hydration sensing technology.

\section{Materials and Methods}

\subsection{Monte Carlo Modeling of Optical Interactions with Porcine Skin}

A Monte Carlo model of optical interaction with porcine skin was developed and executed at 970,1200, and $1450 \mathrm{~nm}$. Typically, the thickness of porcine stratum corneum varies between $20-26 \mu \mathrm{m}$ and that of epidermis varies between $30-140 \mu \mathrm{m}$, with dermis, the thickest skin sublayer, varying between $0.6-3 \mathrm{~mm}$ [30]. Considering the dermal to epidermal thickness ratio to be 10:1 [30], and the total thickness of $1.25 \mathrm{~mm}$, the thickness of the three sublayers were calculated as-stratum corneum $(0.025 \mathrm{~mm})$, epidermis $(0.113 \mathrm{~mm})$, and dermis $(1.112 \mathrm{~mm})$. The simulated tissue volume had a semi-infinite width. The stratification of the simulated tissue volume is illustrated in Figure 2.

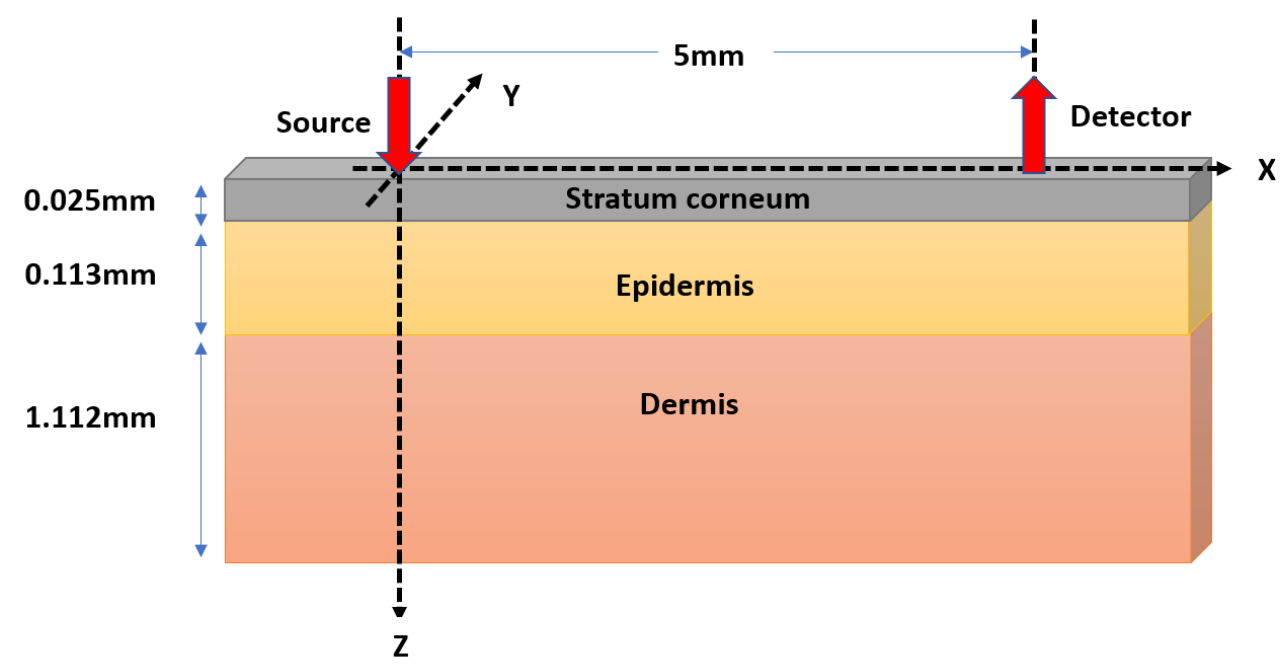

Figure 2. Schematic of the Monte Carlo model. The semi-infinite tissue volume with the finite thickness of $1.25 \mathrm{~mm}$ consisting of three layers are presented in the 3D Cartesian coordinate geometry. The optical source (red downward arrow) is simulated at the origin of the coordinate system, and the detector (red upward arrow) is simulated at a distance of $5 \mathrm{~mm}$ from the source.

The scattering in epidermis and dermis are governed by keratin and collagen fibers, respectively. The scattering coefficients of keratin and collagen are similar [31]. The 
scattering coefficient of skin at 970, 1200, and $1450 \mathrm{~nm}$ are $28.64,26.72$, and $24.83 \mathrm{~mm}^{-1}$, respectively [31,32]. While preparing the samples, blood was removed from the tissue so that the only absorber present in the tissue was water. The absorption properties of the stratum corneum and epidermis were calculated using the formula of baseline tissue absorption coefficient [29]. Originally, this equation was developed for human skin, however, in the absence of blood, the baseline tissue properties, which depend on the structural architecture of porcine and human skin, are similar [30].

In the model, the volumetric distribution of the baseline tissue and water absorption coefficient were considered for calculating the dermis absorption coefficient [29]. The volume fraction in the final measured (i.e., dehydrated) tissue sample was considered to be 0 , and then the hydrated tissue volume was simulated by adding up the desorbed water contents in the reverse order. Table 1 illustrates the water correlation of the measured weight and the volume fraction used in Monte Carlo model, and the details of the dermal absorption coefficients at the sensor wavelengths. The absorption properties of the tissue layers, on the other hand, vary with wavelengths and the water concentration. The effective absorption coefficients were calculated utilizing the volumetric absorbance distribution of the absorbers present in the tissue layers. The blood volume distribution was considered homogeneous in the dermis, and the dermal water contents were varied in 12 steps as mentioned before. The effective absorption coefficient of the dermis $\left(\mu_{a_{\text {dermis }}}\right)$ was calculated utilizing the following equation:

$$
\mu_{a_{\text {dermis }}}(\lambda)=V_{w_{\text {dermis }}} \times \mu_{a_{\text {water }}}(\lambda)+\left(1-V_{w_{\text {dermis }}}\right) \times \mu_{a_{\text {baseline }}}(\lambda)
$$

where $V_{w_{\text {dermis }}}$ represents the volume fraction of water at the three consecutive layers; $\mu_{a_{\text {water }}}, \mu_{a_{\text {baseline }}}$ represent the wavelength-dependent absorption coefficients of water and baseline tissue. A baseline absorption at $5 \%$ and $20 \%$, respectively, was discussed in our previous publication [29]. With this consideration, at 970, 1200, and $1450 \mathrm{~nm}$, the stratum corneum absorption coefficients were $0.0149,0.0074$, and $0.004 \mathrm{~mm}^{-1}$; and the epidermal absorption coefficients were $0.0801,0.1623$, and $4.2934 \mathrm{~mm}^{-1}$, respectively.

Table 1. Water volume fraction in dermis $\left(V_{w}\right)$ and dermal absorption coefficient $\left(\mu_{a_{\text {dermis }}}\right)$ used in the Monte Carlo model corresponding to the measured weight of the tissue.

\begin{tabular}{lcccc}
\hline \multirow{2}{*}{ Weight (g) } & $\boldsymbol{V}_{\boldsymbol{w}_{\text {dermis }}}$ & \multicolumn{3}{c}{$\boldsymbol{\mu}_{\boldsymbol{a}_{\text {dermis }}}\left(\mathbf{m m} \mathbf{m}^{-1}\right)$} \\
\cline { 3 - 5 } & & $\mathbf{9 7 0} \mathbf{~ n m}$ & $\mathbf{1 2 0 0} \mathbf{~ n m}$ & $\mathbf{1 4 5 0} \mathbf{~ n m}$ \\
\hline 1.8244 & 0 & 0.0149 & 0.0074 & 0.0040 \\
1.8567 & 0.0323 & 0.0289 & 0.0408 & 0.9276 \\
1.8994 & 0.075 & 0.0475 & 0.0849 & 2.1487 \\
1.9147 & 0.0903 & 0.0541 & 0.1006 & 2.5862 \\
1.9422 & 0.1178 & 0.0661 & 0.1291 & 3.3726 \\
1.9724 & 0.148 & 0.0793 & 0.1602 & 4.2362 \\
2.0031 & 0.1787 & 0.0926 & 0.1920 & 5.114 \\
2.0187 & 0.1943 & 0.0994 & 0.2081 & 5.5602 \\
2.0417 & 0.2173 & 0.1094 & 0.2318 & 6.2179 \\
2.0513 & 0.2269 & 0.1136 & 0.2417 & 6.4924 \\
2.0652 & 0.2408 & 0.1196 & 0.2560 & 6.8899 \\
2.0833 & 0.2589 & 0.1275 & 0.2747 & 7.4075 \\
\hline
\end{tabular}

In a reflectance sensor geometry, an optical source illuminating a Gaussian beam of $0.5 \mathrm{~mm}$ radius and a circular detector with $0.4 \mathrm{~mm}$ radius were simulated; the source and detector being separated by a distance of $5 \mathrm{~mm}$. Detailed steps of the Monte Carlo simulation and the geometry of the model can be found in our previous publication [29]. A large number of photon packets $\left(\sim 10^{9}-10^{10}\right)$ were computed in order to obtain a high level of accuracy. A 64-bit operating system with an installed memory of 24 GB and an Intel Xeon CPU (2.40 GHz, two processors, Intel, Santa Clara, CA, USA) was dedicated for the simulation. The MATLAB (Mathworks, Inc., Natick, MA, USA) platform was 
chosen for coding and a multi-thread programming environment was used for facilitating the simulation.

\subsection{Design and Development of a Hydration Sensor}

The general system structure of the multi-wavelength sensor is illustrated by the block diagram in Figure 3. The system is comprised of two separate modules; the probe which contains the light sources, detection, and amplification, and the main module that incorporates analogue to digital conversion, current sources, and USB connection for data transfer. A detailed description of the sensor can be found in [33].

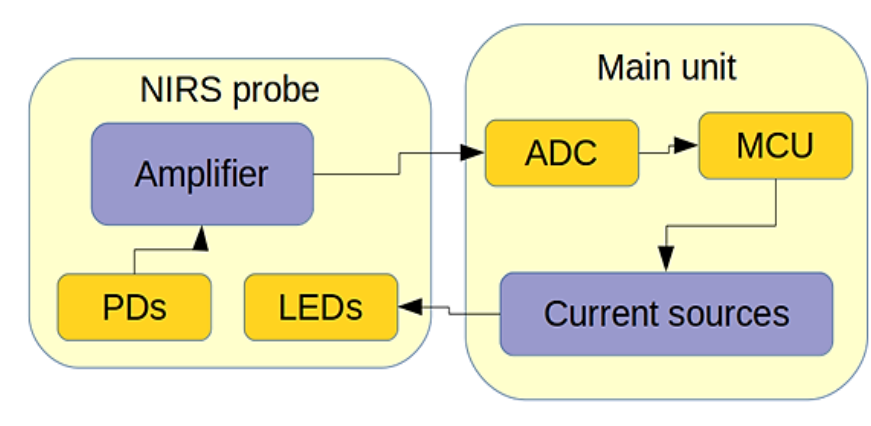

(a)

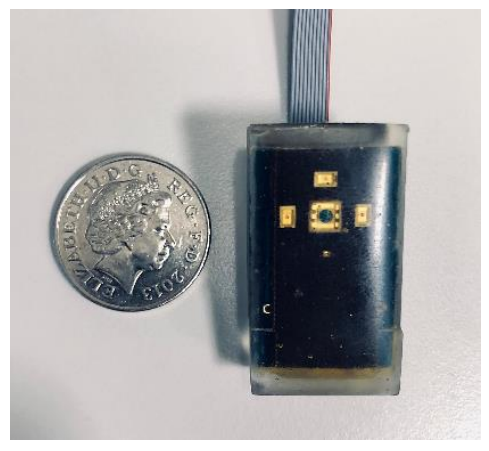

(b)

Figure 3. (a) Hydration sensor system structure, and (b) the hydration sensor probe.

\subsection{In Vitro Experiment}

The study was approved by the Senate Research Ethics committee at City, University of London prior to experiments. Experimental setup was designed to simultaneously record the optical absorbance using the Lambda 1050 Spectrophotometer (PerkinElmer Corp, Waltham, MA, USA) and the developed hydration sensor. The reference values for water content were recorded using a TR-24 electronic precision balance (Denver Instrument $\mathrm{GmbH}$, Göttingen, Germany).

A sample of porcine skin was acquired from an abattoir immediately after slaughtering. The sample was then cut using a scalpel into a $4 \mathrm{~cm} \times 6 \mathrm{~cm}$ rectangular shapes of roughly 1-1.5 mm thickness, and then placed inside an environmental chamber (Model: KMF 115, Binder $\mathrm{GmbH}$, Tuttlingen, Germany) at $96 \%$ relative humidity (RH) and $25^{\circ} \mathrm{C}$ for $48 \mathrm{~h}$. This ensured that maximum hydration levels were reached.

In order to mimic the natural dehydration process through the outermost later of the stratum corneum, customized sample containers were designed and 3D-printed with a single exposure side that isolated the internal sample and ensured that the desorption process occurred only through the outermost layer of the skin.

Once fully hydrated, the skin sample was removed from the chamber and fitted inside the customized container before being placed on the analytical balance. The weight of the container was recorded prior to the insertion of the skin sample, and this value was later deducted from weight recordings and used to calculate values of dermal water content. The probe of the hydration sensor and the fiber optic probe connected to the Lambda 1050 spectrophotometer (PerkinElmer, Waltham, MA, USA) were both placed on the exposed surface of the skin sample and clamped in place for the remainder of the experiment (Figure 4). 


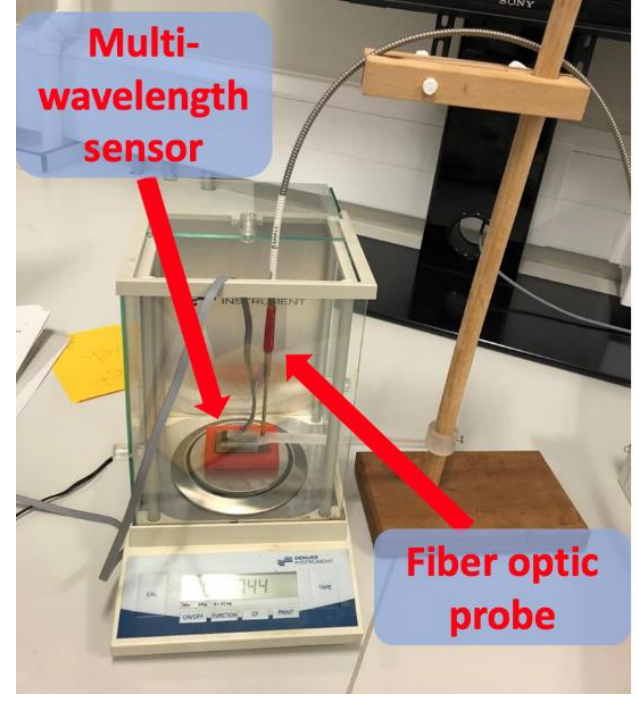

(a)

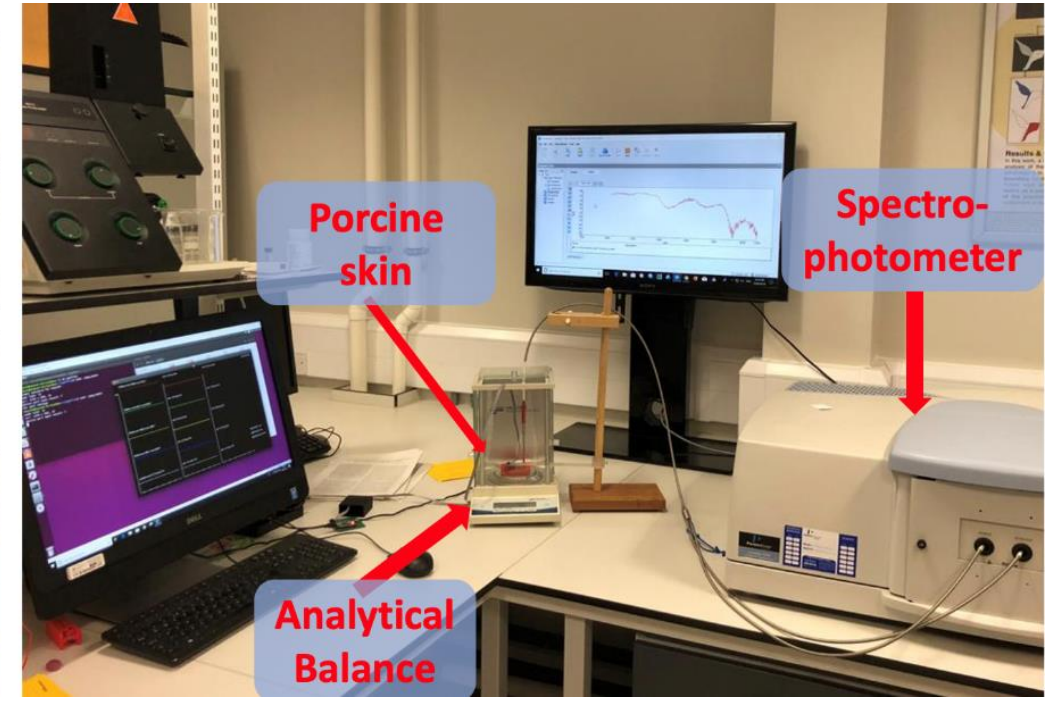

(b)

Figure 4. In vitro experiment setup. (a) The placement of the fiber optic probe and the designed sensor on the sample. (b) The connection of the probes to lambda 1050 and PCs.

Once the set up was complete, weight and optical measurements were initiated, and measurements were made over a 3-h period as the sample underwent desorption.

At twenty-four hours from the start of the experiment and when the specimen was found to be fully dry, a final weight measurement was recorded, reading $1.1183 \mathrm{~g}$. The water content was then approximated by deducting this dry weight value from all weight measurements.

\subsection{Instrument Settings and Data Analysis}

During the 3-h desorption process, data collection was performed in the following way:

1. Intermittent weight measurements were recorded every $20 \mathrm{~min}$, enabling the calculation of dermal water content.

2. DC optical reflectance readings were recorded using the novel hydration sensor at every $30 \mathrm{~s}$ throughout the 3 -h desorption process.

3. A total of 40 reflectance spectra in the range of $872-2100 \mathrm{~nm}$ were collected in $3 \mathrm{~h}$ (every $4.5 \mathrm{~min}$ ). Spectral acquisition was performed using the following instrument settings; InGaAs detection between 872 and $1800 \mathrm{~nm}$, and PbS between 1800 and $2100 \mathrm{~nm}$. The gain and response time for the InGaAs and PbS detectors were set to $3 / 0.2 \mathrm{~s}$ and $1 / 0.2 \mathrm{~s}$, respectively. The attenuator settings were set to $1 \%$ for the reference beam and $100 \%$ for the sample compartment. This was done in order to reduce noise for high absorption values. Furthermore, an initial baseline correction of $100 \%$ transmittance and $0 \%$ absorption was also added.

The efficacy and performance of the developed sensor in measuring dermal water content was analyzed through direct comparison of optical output against the Lambda 1050 spectrophotometer. In this case, gravimetric measurements, obtained from weight measurements using an analytical balance, served as the ground truth of water content, and reflectance spectra acquired with the high-end spectrophotometer were used to benchmark optical measurements. Hence, the analysis evaluated the extent to which the same level of accuracy can be obtained using a portable multi-wavelength optical sensor, and consisted of the following steps:

1. Benchmarking optical measurements of water content with full-range spectra: The full-range optical spectra obtained via Lambda 1050 were preprocessed and subsequently fit to the interpolated weight measurements as a proxy to skin water content. 
2. Investigations into the consistency of output readings from the novel skin hydration sensor and the benchtop spectrophotometer.

3. Development of a multivariate model to predict dermal water content using the skin hydration sensor, the results of which are compared to the Lambda 1050.

\section{Results}

\subsection{Monte Carlo Characterization of the Developed Sensor}

Simulated light-tissue interaction profiles at 970, 1200, and $1450 \mathrm{~nm}$ are presented in Figure 5. As shown in Figure 5a-c, no significant change is visible in the interaction profile as the scattering coefficient values do not vary significantly within the three wavelengths. Optical pathlength, which depends on the scattering coefficient of the tissue, does not vary with the water concentration. The mean depth (i.e., the characteristic depth) penetrated by photons at all three wavelengths are $<1 \mathrm{~mm}$.

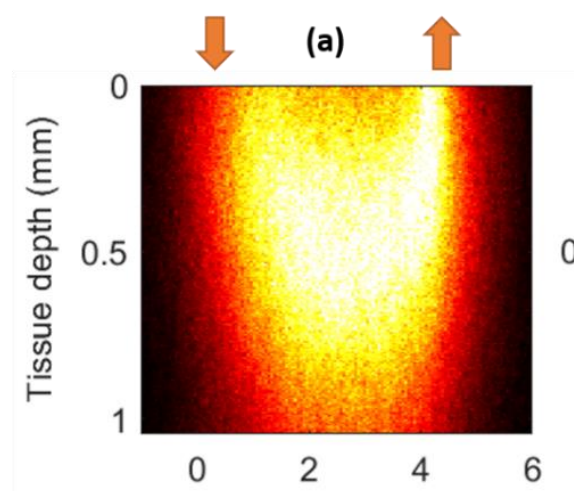

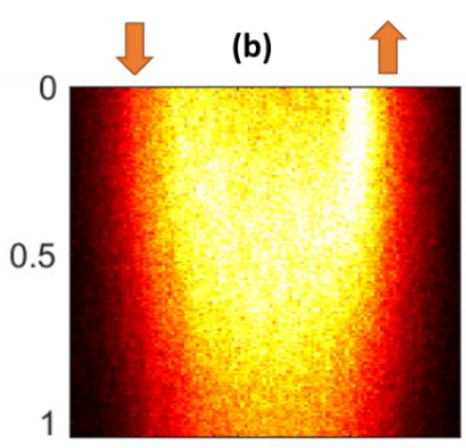

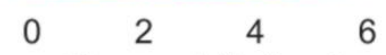

Tissue width $(\mathrm{mm})$
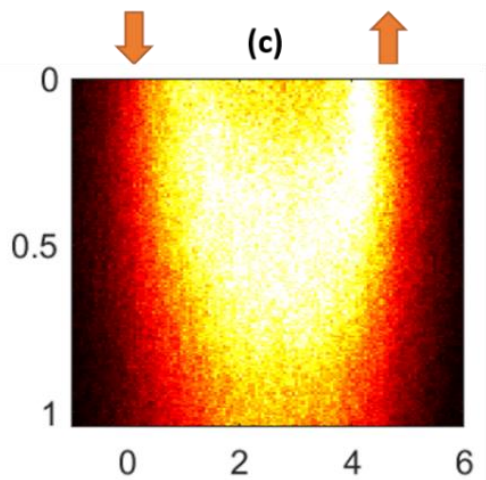

$N_{\max }$
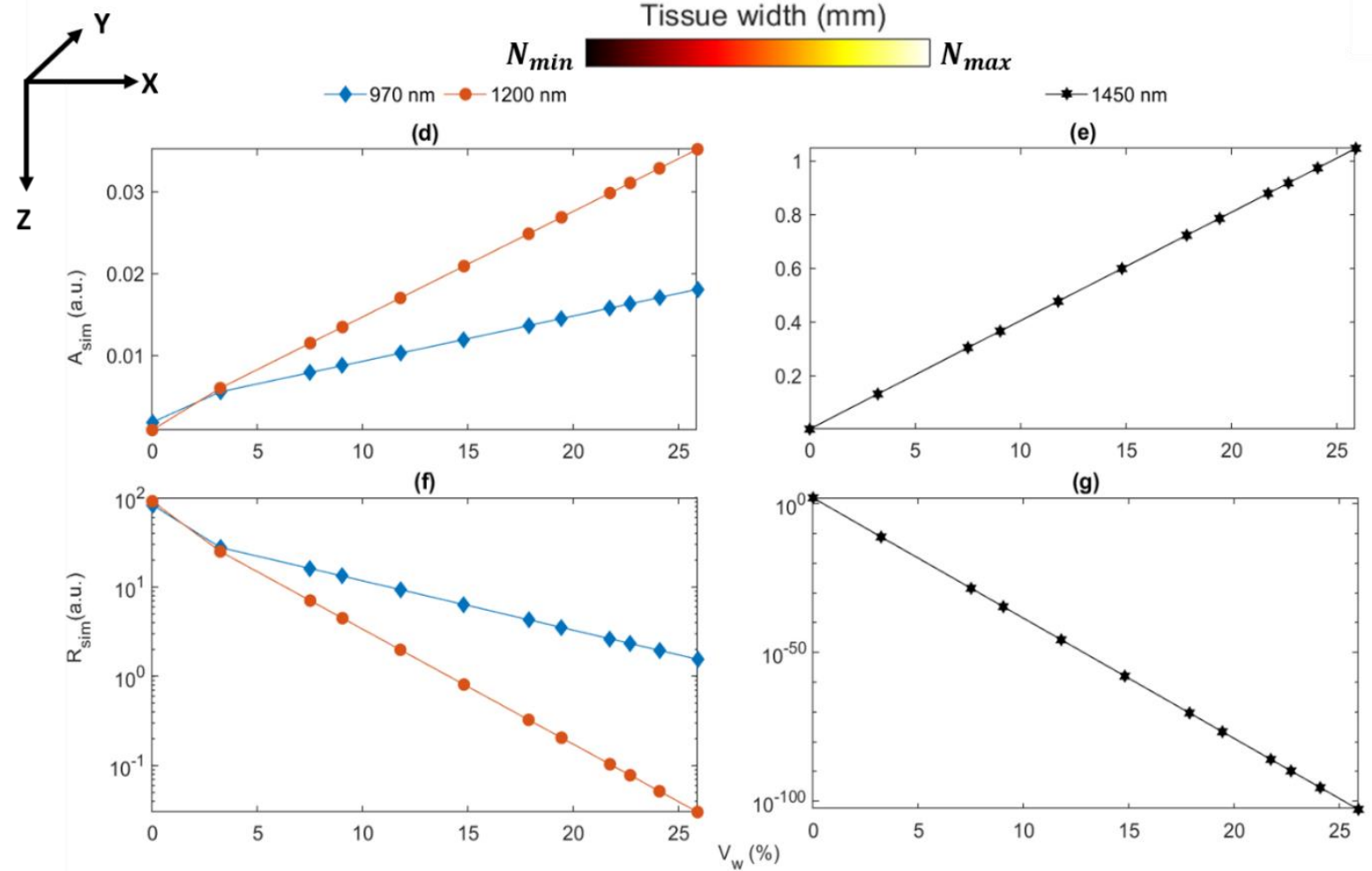

(g)

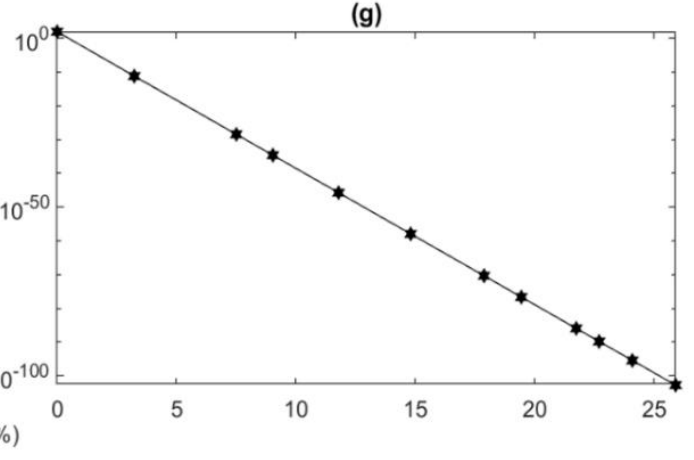

Figure 5. Monte Carlo simulation results at the wavelengths 970, 1200, and $1450 \mathrm{~nm}$. The light-tissue interaction profiles are shown in $(\mathbf{a}-\mathbf{c})$. In the reflectance sensor geometry, the source (upward red arrow) and the detector (the downward red arrow) are separated by a distance of $5 \mathrm{~mm}$. The simulations were carried out with the maximum hydrated skin at all wavelengths. Tissue depth and width are presented along the $z$ - and $x$-axis, respectively. The variations in the simulated absorbance Asim and reflectance Rsim (presented in the logarithmic scale) with the increasing dermal water volume $V w$ (expressed in percentage) are shown at 970 and $1200 \mathrm{~nm}$ in $(\mathbf{d}, \mathbf{f})$, and at $1450 \mathrm{~nm}$ at $(\mathbf{e}, \mathbf{g})$, respectively. 
Shown in Figure $5 \mathrm{~d}-\mathrm{g}$ are the absorbance (Asim) and reflectance (Rsim) which were simulated at the three wavelengths for the 12 different blood volumes (volume fraction $X$ $100 \%$ ). The results for $1450 \mathrm{~nm}$ are shown separately to avoid improper scaling issues. As apparent from these figures, tissue absorbance increases with an increase in water volume. This is true for all simulated wavelengths and indicated by a significant positive slope at $1450 \mathrm{~nm}$ which exhibits a strong water absorbance band. Both the linear increase in absorbance and the exponential decay in intensity (shown as linear plots in the logarithmic scale) resulting from an increase in water concentration are shown to obey the rules of the Beer-Lambert law.

Moreover, changes in absorbance resulting from variations in dermal water concentration were simulated. The baseline stratum corneum and epidermis water concentration were considered based on our previous work [29]. Recent studies have demonstrated the concentration variation of water in stratum corneum towards dermis [14]; incorporation of such detailed parameters may immensely improve the current model. Nevertheless, both simulated and experimental data relating to absorbance and reflectance are highly comparable and demonstrate the prevalence of dermal absorbance in porcine skin hydration measurements.

\subsection{Benchmarking Optical Measurements of Weight with Full-Range Spectra}

In the first step of benchmarking optical measurements of weight with full-range spectra, the acquired diffuse reflectance data were denoised using a Savitzky-Golay filter with the polynomial order of two and the window length of 71 . To account for multiplicative scattering correction (MSC), extended MSC (EMSC) was also performed with quadratic correction factor. Subsequently, the reflectance spectra are min-max normalized and transformed to absorbance spectra with log transformation (Figure 6a).

$$
A \propto \log \left(\frac{1}{R}\right)
$$

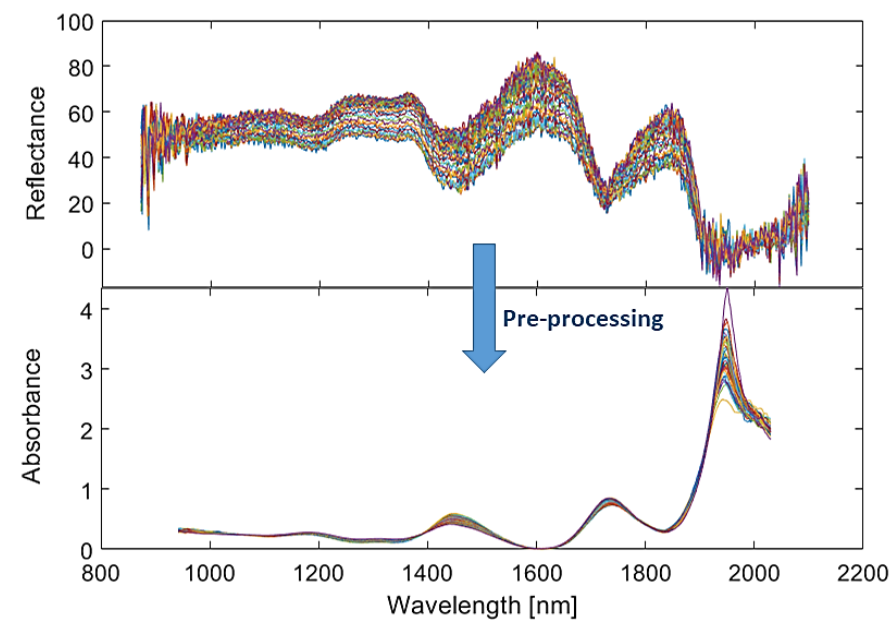

(a)

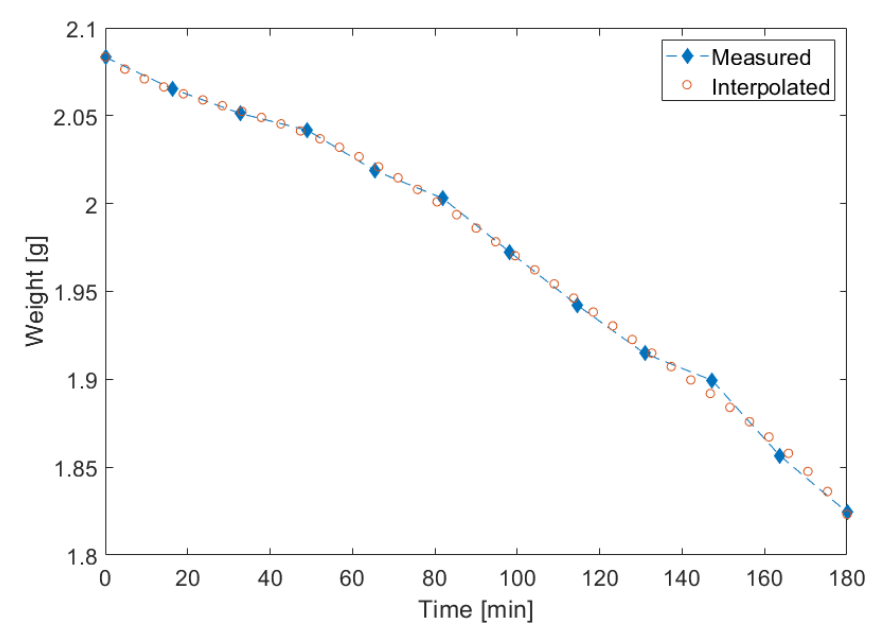

(b)

Figure 6. (a) The preprocessed spectra. (b) Interpolation of weights from the initial measurements.

The resulting absorption spectra (Figure 6a) shows similar bands expected in a typical spectrum of porcine skin such as that presented earlier in Figure 1, dominated by overtone and combination bands of water at 1450 and $1920 \mathrm{~nm}$, as well as the blood combination and overtone bands of $\mathrm{CH}$ bonding that occur between 1730 and $1760 \mathrm{~nm}$, the latter of which appears to be resolved into a single band in the region of 1730-1760 nm. Spectral changes related to changes in water content are also evident as quantitative changes in 
absorption at the relevant bands and as peak shifts in the 1730-1760 $\mathrm{nm}$ region. The latter can be attributed to nonlinear changes at the 1730 and $1760 \mathrm{~nm}$ bands of $\mathrm{CH}$ bonding.

Prior to multivariate modeling, it was necessary to harmonize the time of weight readings and spectral acquisition. To address this, a polynomial of order of five was fit to the weight readings; the weight value pertaining to each spectrum was interpolated from this curve (Figure 6b). Subsequently, a partial least squares (PLS) model with five components was used to regress the interpolated weight values against the optical spectra. The number of components were selected based on the elbow point of the predict residual error sum of squares (PRESS) plot and the performance of the model was evaluated with leave-one-out cross-validation. This led to an RMSECV of $0.0149 \mathrm{~g}$ in the estimation of water content and a coefficient of determination of 0.9590 , showing highly accurate estimates.

The results show a decrease in both weight and spectral absorption over the desorption period, where the decrease in absorption is more distinguishable at the relevant bands of 1450, $1940 \mathrm{~nm}$, and between 1730 and $1760 \mathrm{~nm}$. Thus, both weight and absorption decreased as the dermal water content lessened, and wavelength shifts occurred in the region of $1730-1760 \mathrm{~nm}$, moving from 1735 to $1740 \mathrm{~nm}$ in a manner concurrent with the lessening of water content.

\subsection{The Assessment of Agreement between the Developed Sensor and Lambda 1050 Spectrophotometer}

The optical reflectance values obtained from each LED were first converted to absorbance using the same method that was applied on diffuse reflectance spectra. Due to a mismatch between the time of spectral data acquisition and the more frequent readings of the sensor, a polynomial of order five was fitted to the absorbance readings of the developed sensor for each wavelength, and the absorbance values, corresponding to the time of spectral acquisition, were interpolated.

A linear regression of the form

$$
A_{x}^{\text {Lambda1050 }}=\beta_{0}+\beta_{1} A_{940 \mathrm{~nm}}^{M W S H}+\beta_{2} A_{x}^{M W S H},
$$

where $A$, denotes absorbance, its superscript denotes the device, and the subscript denotes the wavelength. The developed multi-wavelength skin hydration sensor is abbreviated to MWSH. The absorbance pertaining to the wavelength of $940 \mathrm{~nm}$ for the developed sensor is included in all three regressions (i.e., $x=1450,1200$, and $970 \mathrm{~nm}$ ) for baseline correction. Baseline correction for the optical spectra was performed in the preprocessing step as described above. Table 2 summarizes the results.

Table 2. Statistical analysis of the agreement between the absorbance readings from the developed sensor and lambda 1050. Each absorbance reading from Lambda 1050 is separately regressed on the corresponding absorbance values from the developed sensor. The absorbance values for the wavelengths of $940 \mathrm{~nm}$ is included in all regression models for baseline correction.

\begin{tabular}{ccc}
\hline Wavelength $(\mathbf{n m})$ & Regression Coefficient $\boldsymbol{\beta}_{2}{ }^{1}$ & Adjusted Coefficient of Determination $\left(\boldsymbol{R}^{2}\right)$ \\
\hline 1450 & $1.30(0.078)^{*}$ & 0.993 \\
1200 & $1.15(0.313)^{* *}$ & 0.91 \\
970 & $0.13(0.004)^{* * *}$ & 0.837 \\
\hline
\end{tabular}

${ }^{1}$ The standard error is included in brackets. The statistically significant estimates with $p$-values of less than $0.01,0.001$, and 0.0001 are identified with ${ }^{* * *}$, and ${ }^{* * *}$, respectively.

From Table 2 it can be seen that, after correcting for baseline variations, the absorbance values of the developed sensor were highly consistent with the Lambda 1050. In addition, as expected, optical measurements at the $1450 \mathrm{~nm}$ band were more sensitive to variations in water content, and, as a result, outputs from both the developed sensor and spectrophotometer show a significantly higher level of agreement at this wavelength. 


\subsection{Estimation of Water Content and Comparison with Lambda 1050}

Furthermore, a multiple linear model was developed to map the absorbance readings of the developed sensor to water content. The performance of the model was evaluated using leave-one-out cross-validation, and the results were compared with two models developed from diffuse reflectance spectra; the first model uses the complete spectrum (described in part a), whilst the second model uses only the four identified wavelengths. The results are demonstrated in Table 3 .

Table 3. The comparison of the predictive performance of the developed skin hydration sensor with models trained on Lambda 1050 spectra. The first Lambda 1050 model uses the whole spectrum; the second Lambda 1050 model is a multiple linear regression trained on the absorbance readings for wavelengths $940,970,1200$, and $1450 \mathrm{~nm} . R_{C V}^{2}$ denotes the coefficient of determination for the predicted values in the leave-one-out cross-validation.

\begin{tabular}{ccc}
\hline Model & RMSECV (g) & Coefficient of Determination $\left(R_{C V}^{2}\right)$ \\
\hline Skin Hydration Sensor & 0.0038 & 0.9975 \\
Lambda 1050 full spectrum (\#LVs $=5)$ & 0.0149 & 0.9590 \\
Lambda 1050 4 wavelengths & 0.0183 & 0.9429 \\
\hline
\end{tabular}

Comparing the performance of the four-wavelength model and the full spectrum model trained using diffuse reflectance spectra shows only a marginal benefit in the inclusion of additional wavelengths besides the four identified wavelengths. Therefore, the four identified wavelength can adequately-almost equally-capture variations in water content. The table also clearly demonstrates that the developed sensor can not only accurately predict the concentration of water, but it also outperforms the high-end spectrophotometer that was used as a benchmark for optical estimation. The is likely due to the denoising effects of sufficient time-averaging in the sensor. The skin hydration sensor delivers rapid measurements of optical reflectance with the sampling rate of $100 \mathrm{~Hz}$. Subsequently, using an exponential moving average filter, the readings are averaged and a reflectance value is obtained every $30 \mathrm{~s}$. In the spectrophotometer, since the average duration of each scan is about three minutes, the same level of time-averaging cannot be performed; here each spectrum is the average of only three scans. Therefore, denoising is primarily achieved through the application of the Savitzky-Golay filter, which given the minute water-induced variation of the reflectance profile, and the presence of scattering and measurement noise (Figure 5), leads to an inferior predictive performance.

\section{Discussion and Conclusions}

An optical, multi-wavelength skin hydration sensor was designed and developed to provide a compact, continuous, inexpensive, and reliable alternative to electrical capacitancebased technologies. The design leverages three water-sensitive wavelengths $(970,1200$, and $1450 \mathrm{~nm}$ wavelengths) and a water-insensitive wavelength $(940 \mathrm{~nm})$ to estimate the concentration of water in tissue.

Initial sensor evaluation was carried out using the Monte Carlo method, where an in-silico model of porcine skin was developed and explored at the selected wavelengths. The assumptions in the Monte Carlo model regarding the optical properties and thickness were based on the limited available literature. With the careful choice of the simulation parameters, the in-silico model was successfully implemented for the evaluation of the in vitro results and a comprehensive assessment of the light-tissue interactions at the three near-to-mid infrared wavelengths.

Simulation results were complemented by in vitro experiments on porcine skin to validate the design and evaluate the accuracy of its estimates. In this experiment, porcine skin underwent a desorption test, while optical measurements were acquired with the sensor and a high-end spectrophotometer. Gravimetric tests were used as a reference of porcine dermal water content. First, it was shown that the measurements of the skin hydration sensor were highly consistent with that of the high-end spectrophotometer. 
Secondly, we compared the performance of multivariate models developed based on the sensor and the high-end spectrophotometer. This demonstrated that the sensor can accurately predict the concentration of water and exceed the prediction accuracy of the high-end spectrophotometer. Since the spectrophotometer has a higher spectral resolution, the higher accuracy of the sensor is likely due to the denoising effect of temporal averaging; made possible by rapid reflectance measurements. Moreover, the wavelength selectivity implemented in the sensor design allows for better estimation of dermal water content as it utilizes key water absorption bands. For in vivo applications, this will permit acquisition of water-specific information with minimal influence of other biological absorbers. Future work will focus on establishing appropriate algorithms for sensor calibration in the absence of reference gravimetric data.

An accurate, low-cost, continuous, wearable skin hydration sensor can significantly improve disease management in diabetes mellitus, hypothyroidism, malnutrition, and atopic dermatitis. It can also bring about new opportunities in the wellbeing and skin health industry such as product evaluation of cosmetic formulations and skin aging. Our findings suggest that optical technologies may be the key to achieving this aim, and future efforts will focus on further assessment of the feasibility, reliability, and practical limitations of the developed sensor.

Author Contributions: Conceptualization, M.Q. and M.M.; methodology, M.R., M.M., and S.C.; validation, M.Q. and P.A.K.; formal analysis, M.M. and S.C.; investigation, P.A.K.; writing-original draft preparation, M.M., M.R., M.Q., and S.C.; writing—review and editing, M.M. and M.Q.; visualization, M.M. and S.C.; supervision, M.Q. and P.A.K.; project administration, P.A.K. All authors have read and agreed to the published version of the manuscript.

Funding: This research received no external funding.

Institutional Review Board Statement: The study was approved by the Senate Research Ethics committee at City, University of London prior to experiments.

Informed Consent Statement: Not applicable.

Data Availability Statement: Not applicable.

Conflicts of Interest: The authors declare no conflict of interest.

\section{References}

1. Madison, K.C. Barrier function of the skin: “La Raison d'Être” of the epidermis. J. Investig. Derm. 2003, 121, 231-241. [CrossRef] [PubMed]

2. Baroni, A.; Buommino, E.; De Gregorio, V.; Ruocco, E.; Ruocco, V.; Wolf, R. Structure and function of the epidermis related to barrier properties. Clin. Derm. 2012, 30, 257-262. [CrossRef] [PubMed]

3. Verdier-Sévrain, S.; Bonté, F. Skin hydration: A review on its molecular mechanisms. J. Cosmet. Dermatol. 2007, 6, 75-82. [CrossRef] [PubMed]

4. Dobrev, H. Use of Cutometer to assess epidermal hydration. Ski. Res. Technol. 2000, 6, 239-244. [CrossRef]

5. Wiechers, J.W.; Barlow, T. Skin moisturisation and elasticity originate from at least two different mechanisms. Int. J. Cosmet. Sci. 1999, 21, 425-435. [CrossRef] [PubMed]

6. Waller, J.M.; Maibach, H.I. Age and skin structure and function, a quantitative approach (II): Protein, glycosaminoglycan, water, and lipid content and structure. Ski. Res. Technol. 2006, 2, 145-154. [CrossRef]

7. Nguyen, T.T.; Happillon, T.; Feru, J.; Brassart-Passco, S.; Angiboust, J.-F; Manfait, M.; Piot, O. Raman comparison of skin dermis of different ages: Focus on spectral markers of collagen hydration. J. Raman Spectrosc. 2013, 44, 1230-1237. [CrossRef]

8. del Rosso, J.Q.; Levin, J. The clinical relevance of maintaining the functional integrity of the stratum corneum in both healthy and disease-affected skin. J. Clin. Aesthetic Dermatol. 2011, 4, 22-42.

9. Du Plessis, J.; Stefaniak, A.; Eloff, F.; John, S.; Agner, T.; Chou, T.; Nixon, R.; Steiner, M.; Franken, A.; Kudla, I.; et al. International guidelines for the in vivo assessment of skin properties in non-clinical settings: Part 2. transepidermal water loss and skin hydration. Ski. Res. Technol. 2013, 19, 265-278. [CrossRef]

10. Berardesca, E.; Loden, M.; Serup, J.; Masson, P.; Rodrigues, L.M. The revised EEMCO guidance for the in vivo measurement of water in the skin. Ski. Res. Technol. 2018, 24, 351-358. [CrossRef]

11. Farahmand, S.; Tien, L.; Hui, X.; Maibach, H.I. Measuring transepidermal water loss: A comparative in vivo study of condenserchamber, unventilated-chamber and open-chamber systems. Ski. Res. Technol. 2009, 15, 392-398. [CrossRef] 
12. Netzlaff, F.; Kostka, K.H.; Lehr, C.M.; Schaefer, U.F. TEWL measurements as a routine method for evaluating the integrity of epidermis sheets in static Franz type diffusion cells in vitro. Limitations shown by transport data testing. Eur. J. Pharm. Biopharm. 2006, 63, 44-50. [CrossRef]

13. Qassem, M.; Kyriacou, P.A. Review of modern techniques for the assessment of skin hydration. Cosmetics 2019, 6, 19. [CrossRef]

14. Darvin, M.E.; Choe, C.S.; Schleusener, J.; Lademann, J. In vivo non-invasive determination of the water concentration and water bonding properties in the human stratum corneum using confocal Raman microspectroscopy (mini-review). Quantum Electron. 2021, 51, 28. [CrossRef]

15. Sdobnov, A.Y.; Tuchin, V.V.; Lademann, J.; Darvin, M.E. Confocal Raman microscopy supported by optical clearing treatment of the skin-influence on collagen hydration. J. Phys. D Appl. Phys. 2017, 50, 28. [CrossRef]

16. Sdobnov, A.Y.; Darvin, M.E.; Schleusener, J.; Lademann, J.; Tuchin, V.V. Hydrogen bound water profiles in the skin influenced by optical clearing molecular agents-Quantitative analysis using confocal Raman microscopy. J. Biophotonics 2018, 12, e201800283. [CrossRef]

17. Qassem, M.; Kyriacou, P.A. Reflectance near-infrared measurements for determining changes in skin barrier function and scattering in relation to moisturizer application. J. Biomed. Opt. 2015, 20. [CrossRef] [PubMed]

18. Walling, P.L.; Dabney, J.M. Moisture in skin by near-infrared reflectance spectroscopy. J. Soc. Cosmet. Chem. 1989, 40, 151-171.

19. Martin, K. In vivo measurements of water in skin by near-infrared reflectance. Appl. Spectrosc. 1998, 52, 1001-1007. [CrossRef]

20. Martin, K. Direct measurement of moisture in skin by NIR spectroscopy. J. Soc. Cosmet. Chem. 1993, 44, $249-261$.

21. Bodén, I.; Nilsson, D.; Naredi, P.; Lindholm-Sethson, B. Characterization of healthy skin using near infrared spectroscopy and skin impedance. Med Biol. Eng. Comput. 2008, 46, 985-995. [CrossRef] [PubMed]

22. Qassem, M.; Kyriacou, P. Use of reflectance near-infrared spectroscopy to investigate the effects of daily moisturizer application on skin optical response and barrier function. J. Biomed. Opt. 2014, 19, 087007. [CrossRef] [PubMed]

23. Egawa, M. In vivo simultaneous measurement of urea and water in the human stratum corneum by diffuse-reflectance nearinfrared spectroscopy. Ski. Res. Technol. 2009, 15, 195-199. [CrossRef] [PubMed]

24. Egawa, M.; Hirao, T.; Takahashi, M. In vivo estimation of stratum corneum thickness from water concentration profiles obtained with raman spectroscopy. Acta Derm.-Venereol. 2007, 87, 4-8. [CrossRef]

25. Egawa, M.; Arimoto, H.; Hirao, T.; Takahashi, M.; Ozaki, Y. Regional difference of water content in human skin studied by diffuse-reflectance near-infrared spectroscopy: Consideration of measurement depth. Appl. Spectrosc. 2006, 60, 24-28. [CrossRef] [PubMed]

26. Woo, Y.A.; Ahn, J.W.; Chun, I.K.; Kim, H.J. Development of a method for the determination of human skin moisture using a portable near-infrared system. Anal. Chem. 2001, 73, 4964-4971. [CrossRef]

27. Mohamad, M.; Msabbri, A.R.; Matjafri, M.Z. Conceptual design of near infrared spectroscopy instrumentation for skin moisture measurement. In Proceedings of the 2011 IEEE Colloquium on Humanities, Science and Engineering, CHUSER 2011, Penang, Malaysia, 5-6 December 2011; pp. 801-804. [CrossRef]

28. Qassem, M.; Kyriacou, P.A. Comparing the rates of absorption and weight loss during a desorption test using near infrared spectroscopy. Ski. Res. Technol. 2013, 19, 137-144. [CrossRef]

29. Budidha, K.; Kyriacou, P.A.; Chatterjee, S. Investigating the origin of photoplethysmography using a multiwavelength monte carlo model. Physiol. Meas. 2020, 41, 084001. [CrossRef]

30. Summerfield, A.; Meurens, F.; Ricklin, M.E. The immunology of the porcine skin and its value as a model for human skin. Mol. Immunol. 2015, 66, 14-21. [CrossRef]

31. Zamora-Rojas, E.; Aernouts, B.; Garrido-Varo, A.; Saeys, W.; Pérez-Marín, D.; Guerrero-Ginel, J.E. Optical properties of pig skin epidermis and dermis estimated with double integrating spheres measurements. Innov. Food Sci. Emerg. Technol. 2013, 20, 343-349. [CrossRef]

32. Yu, T.; Wen, X.; Tuchin, V.V.; Luo, Q.; Zhu, D. Quantitative analysis of dehydration in porcine skin for assessing mechanism of optical clearing. J. Biomed. Opt. 2011, 16, 095002. [CrossRef] [PubMed]

33. Mamouei, M.; Qassem, M.; Razban, M.; Kyriacou, P.A. Measurement of dermal water content using a multi-wavelength optical sensor. In Proceedings of the Annual International Conference of the IEEE Engineering in Medicine and Biology Society, EMBS, Montreal, QC, Canada, 20-24 July 2020; pp. 4353-4356. [CrossRef] 